\title{
Poster: Martian - Message Broadcast via LED Lights to Heterogeneous Smartphones
}

\author{
Haohua Du ${ }^{1}$, Junze Han ${ }^{1}$, Qiuyuan Huang ${ }^{2}$, Xuesi Jian ${ }^{1}$, Cheng $\mathrm{Bo}^{3}$, Yu Wang ${ }^{3}$, Hongli $\mathrm{Xu}^{4}$ and Xiangyang $\mathrm{Li}^{4}$ \\ ${ }^{1}$ Illinois Institute of Technology , ${ }^{2}$ University of Florida , ${ }^{3}$ University of North Carolina at Charlotte , ${ }^{4}$ USTC , \\ \{hdu4,jhan20,xjian1\}@hawk.iit.edu, xiangyang.li@gmail.com,idfree@ufl.edu, \{cbo1,yu.wang\}@uncc.edu, xuhongli@ustc.edu.cn
}

\begin{abstract}
Visible light communication (VLC) has been shown to have several advantages over traditional wireless communication. We envision a LED-to-smartphone VLC protocol for delivering messages to a group of unsynchronized mobile device receivers. We carefully design and implement our protocol, Martian, which allows smooth communication from the LED lights to a group of camera-enabled mobile devices. Across several phone models, Martian can achieve data rate of about $1.6 \mathrm{kbps}$ even with $N L O S$-light. Our intensive evaluations indicate that, the data rate reaches $4.2 \mathrm{kbps}$ on iPhone 6. This is a significant improvement compared with the $88 \mathrm{bps}$ data rate claimed by state-of-art design.
\end{abstract}

\section{CCS Concepts}

•Networks $\rightarrow$ Mobile networks; Mobile networks;

\section{Keywords}

Visible Light Communication; LED; Smartphone; Heterogeneous

\section{INTRODUCTION}

The wide spread of LED illumination devices have been offering a promising alternative solution of short range wireless communication, known as visible light communication (VLC). However, only a few schemes are proposed for the light-camera communication $[1,2]$ and all of them are designed for device-to-device communications. We design and implement Martian, for lightcamera communication where a message will be broadcasted via LED light and decoded by heterogeneous camera-enabled mobile devices, which could support wide range of fine-grained indoor location based services(Fig 1).

However, several technical challenges have to be addressed to enable Martian work and to achieve significantly improved data rate. 1) uncontrollable captured intensity makes it hard to use the traditional intensity-based modulation (e.g., OOK [1]). 2) unstable non-negligible frame-gap [2] causes unstable transmission loss. 3) unsynchronization between transmitter and receiver when message broadcasting boosts the average delay.

Martian contains two carefully designed layers. The physical layer $(\$ 2.1)$ achieves high throughput communication and handles

Prof. Xiangyang Li is the contact author.

Permission to make digital or hard copies of part or all of this work for personal or classroom use is granted without fee provided that copies are not made or distributed for profit or commercial advantage and that copies bear this notice and the full citation on the first page. Copyrights for third-party components of this work must be honored. For all other uses, contact the owner/author(s).

MobiCom'16 October 03-07, 2016, New York City, NY, USA

(C) 2016 Copyright held by the owner/author(s).

ACM ISBN 978-1-4503-4226-1/16/10.

DOI: http://dx.doi.org/10.1145/2973750.2985257

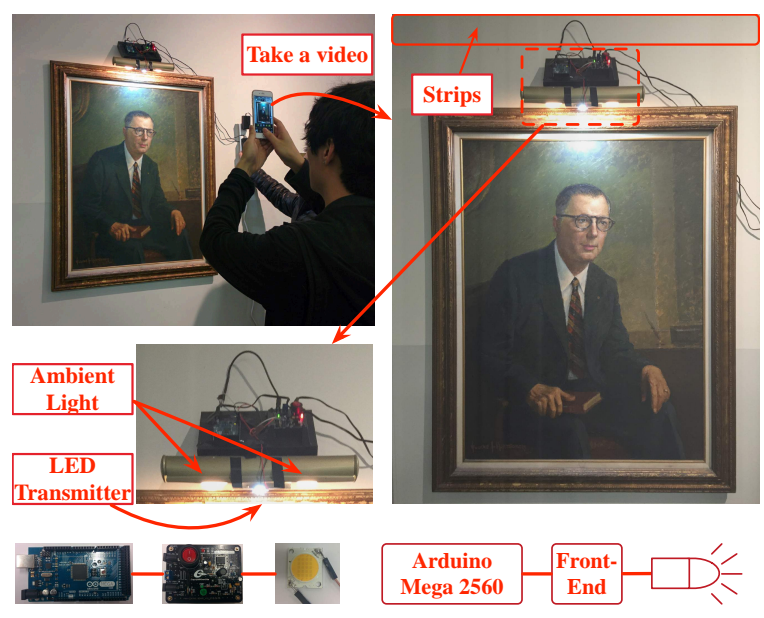

Figure 1: Martin: Broadcasting messages by LED lights, the flashing frequency of transmitter LED light is $4.8 \mathrm{kHz}$ and captured as strips, message.

the extremely unstable channels by $R S$ (Rolling shutter) Manchester Code. The link layer (\$2.2) handles the diversity among frame-gap, frame-time and arriving time, Tabled-based Fountain Code is adopted to improve the achievable data rate. We implement the prototype and test it in a real application. Our extensive evaluations demonstrate that we can achieve a broadcast data rate of $1.61 \mathrm{Kbps}$ at the link/application layer using NLOS light across different phones and locations.

\section{SYSTEM DESIGN}

The structure of Martian contains 6 steps as illustrated in Fig. 2, and works in two different layers - starting from encoding, packaging(in link layer) to modulating(in physical layer) of of transmitter, then recording, demodulating(in physical layer) back to decoding(in link layer) of receiver.

\subsection{PHYSICAL LAYER}

The physical layer is responsible for modulating data into light signals. Traditional Manchester coding strategy changes the light

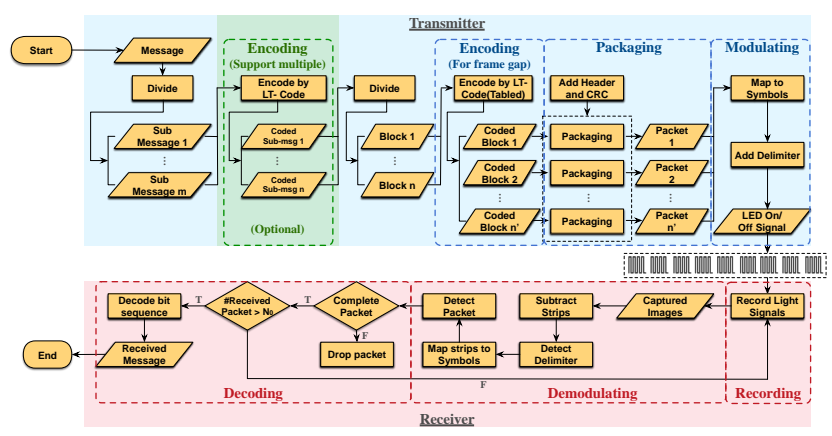

Figure 2: System Flow Overview. 


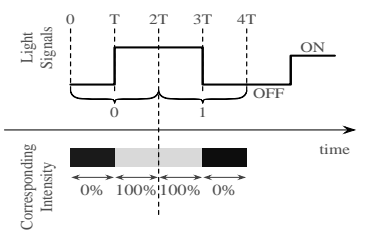

(a)

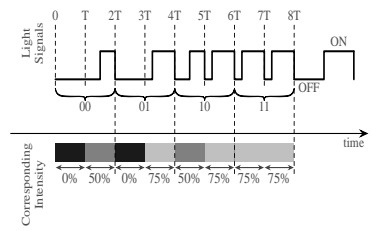

(b)
Figure 3: Modulation strategies: (a) is a naive Manchester modulation and $(b)$ is our proposed modulation.

intensity in two continuous time slots to present a symbol(Fig. 3(a)). However, this approach cannot be adopted directly since both the transmitter and the receiver are unsynchronized. Thus we propose a prefix code to modulate the data bits so that the beginning of each symbol could be identified correctly. We use two time slots to transmit two symbols, and let $i_{1}, i_{2}$ be the intensity of the two continuous slots. Meanwhile, we employ same intensity variation patten between two slots for each symbol $\left(i_{1} \leq i_{2}\right)$, as illustrated in Fig. 3(b), but distinguish bit sequences ' 00 ', '01', ' 10 ', ' 11 ' by different light intensity difference, $i_{2}-i_{1}$. In case the receiver captures the first symbol from a second slot at time $t$, it will drop that symbol and start receiving the coming ones at time slot $t+1$.

\subsection{LINK LAYER}

Our link layer is design to tolerate the symbol and packet loss in the physical layer, then ensure message delivery. There are three major parts of the link layer, encoding, packaging and enabling illumination.

Encoding: We propose a novel table-based fountain code design to reduce the coding information in a packet. Specially, we use a LT-code table $T$, which is hold by the transmitter and the receiver. $T$ is a matrix consists of $t$ vectors of degree $n$, i.e., $T=$ $\left\{\overrightarrow{v_{i}}=\left(v_{i, 1}, \cdots v_{i, n}\right)\right\}$, where $n$ is the number of blocks in a submessage. We determine the appropriate $t$ and $n$ by optimizing the throughput. For a vector $\overrightarrow{v_{i}}$, the $j$-th element $v_{i, j}=1$ if the $j$-th block is used to generate the LT code of the sub-message; $v_{i, j}=0$ otherwise. Instead of transmitting the original sub-message, each packet only includes the LT code of the sub-message and the according index of the vector denoting which blocks are used to generate the LT code. Let $\left|\overrightarrow{v_{i}}\right|=\sum_{j=1}^{n} v_{i, j}$, according to LT-coding scheme, $\left(\left|\overrightarrow{v_{1}}\right|, \cdots,\left|\overrightarrow{v_{n}}\right|\right)$ should be randomly sampled from the robust soliton distribution. We also require that $\forall i_{1}, i_{2} \in[1, \cdots, n]$, the $\left|\sum_{i_{1}=1}^{t} v_{i_{1}, 1}-\sum_{i_{2}=1}^{t} v_{i_{2}, 1}\right|<H$, where $H$ is a predefined threshold. Let $n_{r}$ be the number of packets received for a submessage, then the sub-message can be decoded and recovered if $n_{r}>N_{0}$, where $N_{0}$ is the minimum number of received blocks required to decode in LT-coding scheme.

Packaging: Each packet contains four parts, delimiter, header, message body and parity. Delimiter contains 6 darkest light symbols to indicate the beginning of a packet. A header contains a field representing which sub-message is contained in the packet, and a field representing which blocks are used to generate the LT coding block in the packet. Additionally, parity contains a CRC segment for error detection and correction.

Enabling illumination: We also need to mitigate the flicker to enable a harmonious illumination. If the intensity changes at least once within $1 / 50$ seconds, it would be neglected by human eyes.Thus, we scramble every packet following below algorithm to eliminate flickers.

\section{FEASIBILITY RESULTS}

We implement the modulation process of transmitter and encod-

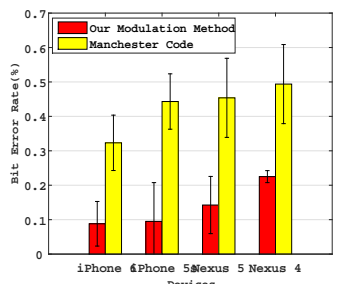

(a) BER: Our v.s. Manchester.

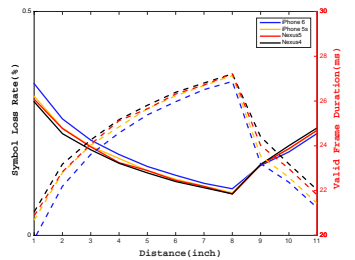

(c) Symbol Loss v.s. Distance.

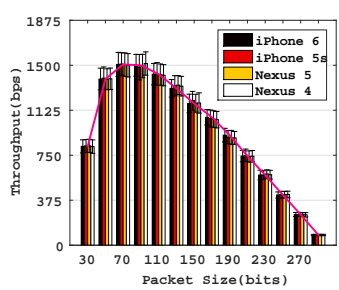

(e) Throughput v.s. P-Size.

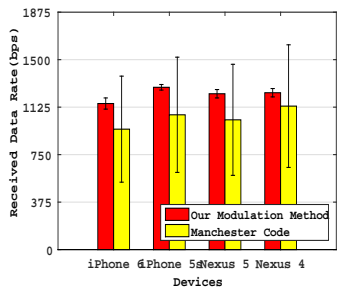

(b) RDR: Our v.s. Manchester.

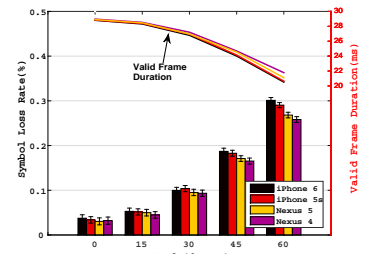

(d) Symbol Loss v.s. Angle.

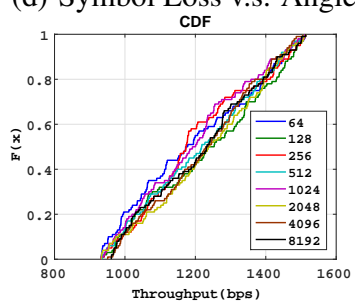

(f) Throughput v.s. T-Size.
Figure 4: Preliminary experiments results.

ing stage on Arduino Mega 2560. And we use iPhone 6, iPhone $5 \mathrm{~S}$, Nexus 5 and Nexus 4 as receiver separately.

Evaluate symbol design in physical layer. Fig 4(a) and Fig 4(b) show how efficiency our modulation scheme can achieve, compared with original Manchester coding scheme from bit error rate(BER) and received data rate(RDR) respectively. The mean and variance of the RDR, and BER of our scheme are much smaller than those of the Manchester scheme since our scheme does better on handling unsynchronized channels.

Estimate environmental impact on physical layer. We examine our physical protocol under various environments. Surprisingly, as shown in Fig. 4(c) and Fig. 4(d), the symbol loss rate $l_{s}$ decreases with the increase of distance/angle initially. However, it will increase when distance/angle is larger than a threshold (about 8 inches in our experiment).

Transmission efficiency in link layer. Both Fig 4(e) and Fig 4(f) show that our throughput is able to achieve $1.54 \mathrm{kbps}$ when packet size(P-Size) is about 70bits or table size(T-Size) is about 2048.

\section{ACKNOWLEDGEMENTS}

This research is partially supported by NSF ECCS-1247944, NSF CMMI 1436786, NSF CNS 1526638, National Natural Science Foundation of China under Grant No. 61520106007, 61428203 and 61572347.

\section{5i] DANAKIS, RENCES}

1] DANAKIS, C., AFGANI, M., Povey, G., Underwood, I. AND HAAS, H. Using a cmos camera sensor for visible light communication. In GC Wkshps, 2012 IEEE (2012), IEEE, pp. 1244-1248.

[2] LeE, H.-Y., Lin, H.-M., WeI, Y.-L., Wu, H.-I., Tsai, H.-M., AND LIN, K. C.-J. Rollinglight: Enabling line-of-sight light-to-camera communications. In MobiSys (2015), ACM, pp. 167-180. 